\title{
Proposed Immunology of the Spontaneous Remission of Leukemia
}

\author{
Behzad Niakan*
}

Pharm. D, USA

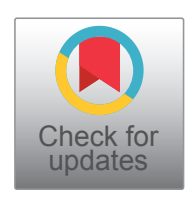

*Corresponding author: Behzad Niakan, Pharm. D., P.O. Box 25817, Los Angeles, Ca. 90025, USA, Tel: (310)-925-2720

\begin{abstract}
Spontaneous remission of leukemia is more likely to occur after anti-biotic treatment of an infection, termination of pregnancy, blood transfusion and host versus graft reaction. All have in common a drop in platelet count followed by a rise in platelet count.

Spontaneous remissions of leukemia may be preceded by a low platelet count, anemia or low hemoglobulin. Also, once spontaneous remission of leukemia occurs there may be a simultaneous rise in platelet count and hemoglobulin.

The drop and rise in platelet count seem to be suggestive of an acute inflammatory response and the acute inflammatory response was possibly the cause of the spontaneous remission of leukemia. It is suggested that the acute inflammatory response that has taken place was initiated while the platelet count was low. There after by passing the immune suppressive effects of the systemic inflammation induced by the leukemia.

Platelets seem to be the medium of the immune-suppressive effect of the systemic inflammation induced by the leukemia. The lowering of activated platelets in patients with leukemia may allow by passing the immune-suppressive effects and tumor promoting effects of activated platelets in leukemia patients and thereafter the activation of the immune system and the removal of leukemia cells. Platelets have an immune-regulatory function effecting both innate immunity and adoptive immunity.

Possibly the low blood oxygen enhanced the acute inflammatory response initiated with a low platelet count. A low Hemoglobin might have made cancer more vulnerable to an immune response since cancer cells are more dependent on iron than normal cells are.
\end{abstract}

\section{Introduction}

Researchers have been baffled by the case reports of the spontaneous remission of leukemia. It may last for a short period, rarely it my last for a prolonged period. Leukemia has many different forms. Some are very aggressive and dangerous. At time these aggressive leukemias spontaneously disappear without any medical intervention. Most of the reported cases of the spontaneous remission of leukemia may last for a short while rarely does the spontaneous remission of leukemia last for a prolonged period.

A study of the case reports of the spontaneous remission of leukemia in the medical literature point out that these spontaneous remissions of leukemia are more likely to occur after an infection particularly after anti-biotic treatment or after the termination of pregnancy particularly if gestational thrombocytopenia is present or after blood transfusions particularly if graft-versus-host reaction occurs. Anti-biotic treatment of infection and blood transfusion and termination of pregnancy and graft versus host reaction may have something in common in activating the immune system in leukemia patients.

Infection (sepsis, etc.) may lower the platelet count after antibiotic treatment the platelet count may rise. Pregnancy may be accompanied with gestational thrombocytopenia and anemia. After termination of pregnancy there is a spontaneous rise of platelet count. Blood transfusion might be needed due to a low platelet count or anemia. During blood transfusion a graft-versus-host reaction may occur which could further lower the platelet count. After blood transfusion a rise in platelet may occur. This suggested rise in platelet count may occur if no precondition such as bone marrow suppression preventing a rise in platelet count (megakaryopoiesis, is the complex process of hematopoietic stem cells developing into platelets).

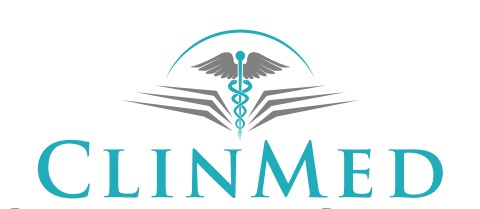

INTERNATIONAL LIBRARY

Citation: Niakan B (2021) Proposed Immunology of the Spontaneous Remission of Leukemia. Int J Cancer Clin Res 8:161. doi.org/10.23937/2378-3419/1410161 
Platelets have an immune-regulatory function effecting innate immunity cells (NK cells, Dendrite cells, etc.) and adoptive immunity. Innate immunity acts as surveillance system to remove damaged cells such as virus infected cells or cancer cells. Dendrite cells present antigen to adoptive immunity and adoptive immunity becomes activated.

Platelets are one of the early responders in an acute inflammatory response. During an acute inflammatory response innate immunity is activated and later adoptive immunity. During an acute inflammatory response the injured tissue regresses to an embryonic state. Later this embryonic tissue differentiates to new tissue and tissue repairs occur. During the acute inflammatory response unhealthy or damaged cells are removed by innate immunity. Malignant tissue is similar to damaged or unhealthy embryonic tissue. Yet, innate immunity is disabled to remove the malignant cells.

\section{Methods}

Different disciplines related to malignant growth were studied such as biology, immunology, neurology, etc. Afterwards a focus was made on the case reports of the spontaneous regression and remission of cancer. Case reports of the spontaneous regression and remission of cancer since the late 1800's was studied. There after the case reports were categorized by tissue of origin and the event preceding the spontaneous remission of cancer. Afterwards focus was made on the case reports of the spontaneous remission of leukemia and the events preceding it. The objective was to find a common denominator among these case reports of the spontaneous remission of leukemia.

\section{Results}

Review of the case reports of the spontaneous remission of leukemia point out that they may occur more commonly after an anti-biotic treatment of an infection or after termination of pregnancy or after blood transfusion or after a host versus graft reaction. The drop and rise in platelet count seem to be the common denominator among them.

Low blood oxygen (low hemoglobin) and fever has also been noted among the reported cases of the spontaneous remission of leukemia. Possibly the low blood oxygen and fever enhances an acute inflammatory response in the presence of a low platelet count.

It seems those spontaneous remissions of leukemia that lasted for a prolonged period were preceded by a low dose of a synthetic corticosteroid. Synthetic corticosteroid such as prednisone, etc. some case reports of prolonged spontaneous remission of solid tumor are also preceded by a low dose of a synthetic corticosteroid.

Any type of cancer may spontaneously remit after anti-biotic treatment of an infection or after termination of pregnancy or after blood transfusion. It is not specific to leukemia.

\section{Discussion}

Spontaneous remission of leukemia more commonly occurs after antibiotic treatment of an infection or after termination of pregnancy or after blood transfusion. What is common between antibiotic treatment of an infection and termination of pregnancy and blood transfusion is a low platelet count and a possible low hemoglobin before the spontaneous remission of leukemia and a simultaneous rise in platelet count and hemoglobin with the spontaneous remission of leukemia. The drop and rise of the platelet count seem to be suggestive of an acute inflammatory response initiated with a low platelet count. Anemia possibly enhances the acute inflammatory response.

Low hemoglobin may have made cancer cells vulnerable to an immune response. since, cancer cells are more dependent on iron than normal cells are.

Spontaneous remission of leukemia is more likely to occur after anti-biotic treatment of an infection [1,2] or after termination of pregnancy [3] or after blood transfusion [4, 7] or after host versus graft reaction [5]. What is common among them is a low platelet count before the spontaneous remission and after the spontaneous remission a rise in platelet count. It seems to be suggestive of an acute inflammatory response. Since, during an acute inflammatory response there is a drop in platelet count followed by a rise in platelet count. During an acute inflammatory response there is a short and temporary inhibition of megakaryopoiesis followed by a rise in platelet count (thrombocytosis).

The systemic inflammation induced by the malignancy may be followed by thrombocytosis and hypercoagulation that disrupt a normal acute inflammatory response. Particularly, thrombocytosis opposing a temporary inhibition of megakaryopoiesis during an acute inflammatory response. Possibly the low platelet count noticed prior to the spontaneous remission of leukemia has negated thrombocytosis and allowed for the temporary inhibition of Megakaryopoiesis.

Megakaryopoiesis is temporary inhibited in response to injury during an acute inflammatory response. Possibly this temporary inhibition of Megakaryopoiesis is needed for innate immunity to recognized damaged cells such as cancer cells and present them to adoptive immunity and afterwards their removal. During malignant growth a state of continuous thrombocytosis induced by the malignancy exists negating the temporary inhibition of Megakaryopoiesis needed for innate immunity to possibly recognized cancer cells.

Since, some of the case reports of spontaneous remissions of leukemia are preceded by a low platelet count one may suggest that the low activated platelet count may have allowed for the temporary inhibition of 
Megakaryopoiesis thereby allowing the recognition of cancer cells by innate immunity.

Furthermore, low activated platelet count may disable (negate) the ability of activated platelets in cancer patients to promote tumor growth such as promoting angiogenesis and tumor neovascularization. In addition, low activated platelet count may disable (negate) the ability of activated platelets in cancer patients to inhibit immune responses against cancer cells such as platelets inhibiting NK cells from removing cancer cells.

It seems Platelets can be activated by a specific inflammatory response either during an acute inflammatory response or during a systemic inflammation induced by the malignancy. Not by both and they seem to be exclusive of one another. If platelets are activated during an acute inflammatory response the immune system removes the malignant cells. If platelets are activated by a systemic inflammation induced by the malignancy the platelets become protective of the malignant tissue. Platelets inhibiting NK cells from removing malignant cells is an example of platelets protecting the malignant tissue. Possibly a function of platelets is to protect the integrity of the tissue that activates it. During an acute inflammatory response due to injury possibly platelets are directing innate immunity cells to remove damaged cells such as virus infected cells or malignant cells. Possibly a low platelet count (possibly the absence of thrombocytosis and hyper-coagulation) as noted in some of the case reports of the spontaneous remission of leukemia may allow for a shift from platelet activation through the systemic inflammation induced by the malignancy to platelet activation through the acute inflammatory response and the removal of the malignancy.

Platelets have an immune-regulatory function. Activated platelets in cancer patient may suppress immune response such as inhibiting NK cells from removing cancer cells [6]. Also, activated platelets in cancer patients may induce tumor growth by promote angiogenesis and tumor neovascularization. It seems to point out a pivotal role of a low platelet count in spontaneous remission of leukemia.

Furthermore, the adrenal may affect an acute inflammatory response. Stress can result in hypercoagulation. A hallmark of cancer is hyper-coagulation. A low dose of Dexamethasone or Prednisone may negate the hyper-coagulation and allow for an acute inflammatory response not hindered by the malignancy.

It appears a combination of low platelet count and fever and low blood oxygen level may have an additive effect in reactivating a suppressed innate immunity in cancer patients. It seems that if a platelet is not low enough, anemia or fever may have a cumulative effect to reactivate a suppressed innate immunity in cancer patients. Low platelet count and low blood oxygen and a fever have in common the induction of dendrite cell maturation.

Possibly the remission of leukemia or any other malignancy might be induced by inducing an acute inflammatory response in the presence of low platelet count (absence of thrombocytosis) if a preexisting condition such as bone marrow suppression is not

Table 1: Spontaneous remission of leukemia.

\begin{tabular}{|c|c|c|c|c|}
\hline & Platelet/L & Hemoglobin/dl & Fever/C & Comments \\
\hline Ref. \# [7] & $98 \times 10(9) / L$ & $10.5 \mathrm{~g} / \mathrm{dl}$ & $40.7^{\circ} \mathrm{C}$ & (reduced megakaryocytes, DIC) \\
\hline Ref. \# [8] & $54 \times 10(9) / L$ & $6.0 \mathrm{~g} / \mathrm{dl}$ & $39.5^{\circ} \mathrm{C}$ & $\begin{array}{l}\text { (reduced megakaryocytes before remission and sufficient after } \\
\text { remission, mechanical ventilation, pneumonia, RBC \& PLT } \\
\text { transfusion) }\end{array}$ \\
\hline Ref. \# [9] & $33 \times 10(9) / L$ & $7.9 \mathrm{~g} / \mathrm{dl}$ & $41.0^{\circ} \mathrm{C}$ & (case \#2, PLT transfusion, mechanical ventilation, pancytope) \\
\hline Ref. \# [10] & $28 \times 10(9) / L$ & $7.1 \mathrm{~g} / \mathrm{dl}$ & $40.5^{\circ} \mathrm{C}$ & (frequent RBC transfusion, pancytopenia, daily fever) \\
\hline Ref. \# [11] & $120 \times 10(9) / L$ & $8.5 \mathrm{~g} / \mathrm{dl}$ & $39.2^{\circ} \mathrm{C}$ & (anti-HLA antibodies, pneumonia, tuberculosis) \\
\hline Ref. \# [12] & $26 \times 10(9) / L$ & $7.7 \mathrm{~g} / \mathrm{dl}$ & $39.0^{\circ} \mathrm{C}$ & (gestational thrombocytopenia) \\
\hline Ref. \# [13] & $27 \times 10(9) / L$ & $5.8 \mathrm{~g} / \mathrm{dl}$ & $38.5^{\circ} \mathrm{C}$ & (case 2 , fever over $38.5^{\circ} \mathrm{C}$, transfusion of RBCs and PLTs) \\
\hline Ref. \# [14] & $28 \times 10(9) / L$ & $7.4 \mathrm{~g} / \mathrm{dl}$ & $38.6^{\circ} \mathrm{C}$ & (gestational thrombocytopenia) (reported as $101.5^{\circ} \mathrm{F}$ ) \\
\hline Ref. \# [15] & $100 \times 10(9) / L$ & $7.6 \mathrm{~g} / \mathrm{dl}$ & $\mathrm{N} / \mathrm{A}$ & (GI septicemia, remission and increased megakaryocytes) \\
\hline Ref. \# [16] & $20 \times 10(9) / L$ & $7.8 \mathrm{~g} / \mathrm{dl}$ & $\mathrm{N} / \mathrm{A}$ & $\begin{array}{l}\text { (gestational thrombocytopenia, blood transfusion PLT, RBC, } \\
\text { Possibly chemotherapy after spontaneous remission) }\end{array}$ \\
\hline Ref. \# [17] & $216 \times 10(9) / L$ & $8.5 \mathrm{~g} / \mathrm{dl}$ & N/A & (Fibrinogen 460 mg/dl, severe anemia) \\
\hline Ref. \# [18] & $64 \times 10(9) / L$ & $9.7 \mathrm{~g} / \mathrm{dl}$ & $\mathrm{N} / \mathrm{A}$ & (Bacterial Pneumonia) \\
\hline Ref. \# [19] & $102 \times 10(9) / L$ & $9.6 \mathrm{~g} / \mathrm{dl}$ & $\mathrm{N} / \mathrm{A}$ & (severe pneumonia, blood transfusion of PLT and RBC) \\
\hline Ref. \# [20] & $17 \times 10(9) / L$ & $5.37 \mathrm{~g} / \mathrm{dl}$ & $\mathrm{N} / \mathrm{A}$ & (infection, persistence fever, transfusion RBCs \& PLTs) \\
\hline Ref. \# [21] & $40 \times 10(9) / L$ & $8.0 \mathrm{~g} / \mathrm{dl}$ & $\mathrm{N} / \mathrm{A}$ & (fever, chills) \\
\hline Ref. \# [22] & $10 \times 10(9) / L$ & $10.4 \mathrm{~g} / \mathrm{dl}$ & N/A & $\begin{array}{l}\text { (gestational thrombocytopenia, prothrombin time } 16.8 \mathrm{sec} \text {; } \\
\text { Thromboplastin time } 24.0 \mathrm{sec} \text {., spontaneous remission } \\
\text { Possibly prior to chemotherapy) }\end{array}$ \\
\hline
\end{tabular}


preventing the rise of platelet count after an acute inflammatory response. Low hemoglobin possibly enhances the acute inflammatory response. a low hemoglobin may make cancer more vulnerable to an immune response $[23,24]$. A Low dose of a synthetic corticosteroid such as prednisone or dexamethasone may enhance the acute inflammatory response and negate the effect of the adrenal.

\section{Caution}

A hypothesis is presented for the spontaneous remission of leukemia. No clinical proof presented for the suggested hypotheses. Medical supervision needed for experimental treatment. Platelet count or blood oxygen level may drop too low. Close monitoring of patient needed.

\section{Disclaimer}

Always seek the advice of your physician or other qualified health provider with any questions you may have regarding a medical condition. Never disregard professional medical advice or delay in seeking it because you have read this study. This is not intended to be a substitute for medical advice, diagnosis or treatment in regards to any patient.

\section{Conflict of Interest}

No conflict of interest.

\section{References}

1. Rashidi A, Fisher SI (2015) Spontaneous remission of acute myeloid leukemia. Leuk Lymphoma 56: 1727-1734.

2. Kostner AH, Johansen RF, Schmidt H, Molle I (2013) Regression in cancer following fever and acute infection. Acta Oncol 52: 455-457.

3. de Mayolo JA, Ahn YS, Temple JD, Harrington WJ (1989) Spontaneous remission of acute leukemia after termination of pregnancy. Cancer 63: 1621-1623.

4. M Mitterbauer, M Fritzer-Szekeres, G Mitterbauer, I Simonitsch, P Knöbl, et al. (1996) Spontaneous remission of acute myeloid leukemia after infection and blood transfusion associated with hypergammaglobulinaemia. Ann Hematol 73: 189-193.

5. Schwarzenberg L, Mathé G, Schneider M, Amiel JL, Cattan A, et al. (1966) Attempted adoptive immunotherapy of acute leukemia by leukocyte transfusions. Lancet 2: 365-368.

6. Kopp HG, Placke T, Schmiedel BJ, Kanz L, Krusch M, et al. (2008) NK cell anti-tumor reactivity is impaired by platelet derived TGF-B through NKG2D down regulation. Blood 112: 2859 .

7. Maywald O, Buchheidt D, Bergmann J, Schoch C, Ludwig W-D, et al. (2004) Spontaneous remission in adult acute myeloid leukemia in association with systemic bacterial infection - case report and review of the literature. Ann Hematol 83: 189-194.

8. Mozafari R, Moeinian M, Asadollahi-Amin A (2017) Spontaneous complete remission in a patient with acute myeloid leukemia and severe sepsis. Case Rep Hematol 2017: 9593750 .
9. Trof RJ, Beishuizen A, Wondergem MJ, Strack van Schijndel RJM (2007) Spontaneous remission of acute myeloid leukemia after recovery from sepsis. Neth $\mathrm{J}$ Med 65: 259-262.

10. Helbig D, Quesada AE, Xiao W, Roshal M, Tallman MS, et al. (2020) Spontaneous remission in a patient with acute myeloid leukemia leading to undetectable minimal residual disease. J Hematol 9: 18-22.

11. Ifrah N, et al. (1985) Spontaneous remission in adult acute leukemia. Cancer 56: 1187-1189.

12. Yalcin A, Aydemir N, Keskin A, Erbay H, Bir F (2002) A case of acute myelomonocytic leukemia in a patient whom refused therapy. The Internet Journal of Hematology 1: 1-4.

13. Zeng Q, Yuan Y, Li P, Chen T (2013) Spontaneous remission in patients with acute myeloid leukemia with $t(8 ; 21)$ or cutnaneous myeloid sarcoma: two case report and review of the literature. Internal Medicine 52: 12271233.

14. Mayolo JN, Ahn YS, Temple JD, Harrington WJ (1989) Spontaneous remission of acute leukemia after the termination of pregnancy. Cancer 63: 1621-1623.

15. Bradley T, Zuquello RA, Aguirre LE, Mackrides N, Chapman $\mathrm{J}$, et al. (2020) Spontaneous remission of acute myeloid leukemia with NFI alteration. Leuk Res Rep 13: 100204.

16. Fukuoka K, Nishikawa K, Mizumoto $\mathrm{Y}$, Shimoyama T, Mikasa K, et al. (1989) [acute myelogenous leukemia developing in pregnancy with complete remission - a case report]. Rinsho Ketsueki 30: 1859-1864.

17. Kizaki M, Ogawa T, Watanabe Y, Toyama K (1988) Spontaneous remission in hypoplastic acute leukemia. Keio J Med 37: 299-307.

18. Takeuchi M, Tamaoki A, Soda R, Takahashi K (1999) Spontaneous remission of large grandular lymphocyte $T$ cell leukemia. Leukemia 13: 313-314.

19. Shimohakamada Y, Shinohara K, Fukuda N (2001) Remission of acute myeloblastic leukemia after severe pneumonia treated with high-dose metylprednisolone. Int J Hematol 74: 173-177.

20. Mabed Md, et al. (2017) Spontaneous remission in acute lymphoblastic leukemia: A case report and review of literature. Hematol \& Transfusion Int J. 4: 4 100-101.

21. Lefrere F, Hermine O, Radford-Weiss I, Veil A, Picard F, et al. (1994) A spontaneous remission of lymphoid blast crisis in chronic myelogenous leukemia flowing blood transfusion and infection. Br J Haematol 88: 621-622.

22. Hoxha SL, Ibishi VA, Brovina A, Hoxha M, Lulaj S (2013) Refusal of treatment for acute leukemia in pregnancy: $A$ case report. J Med Case Rep 7: 148.

23. Ibrahim O, O'Sullivan J (2020) Iron chelators in cancer therapy. Biometals 33: 201-215.

24. Morales M, Xue X (2021) Targeting iron metabolism in cancer therapy. Theranostics 11: 8412-8429. 